Proceedings of SALT 30: 464-484, 2020

\title{
How obligatory irrelevance, symmetric alternatives, and dense scales conspire: The case of modified numerals and ignorance*
}

\author{
Brian Buccola \\ Michigan State University
}

\author{
Andreas Haida \\ The Hebrew University of Jerusalem
}

\begin{abstract}
Buccola \& Haida (2019) explore the consequences of a semantic-pragmatic theory in which relevance is closed under speaker belief (Fox 2016). A primary consequence of this closure condition, they show, is that the Maxim of Quantity commits speakers to expressing their epistemic state about every relevant proposition. We argue that this commitment, dubbed Strong Epistemic Transparency, explains the contrast in ignorance inferences exhibited by non-strict comparative expressions like at least vs. strict ones like more than (hence the class A/B distinction of Nouwen 2010). We also discuss how our analysis might be extended to account for the observations of Cummins, Sauerland \& Solt (2012) and Enguehard (2018) that the modifier more than does not block scalar inferences of round numerals.
\end{abstract}

Keywords: ignorance inferences, scalar implicatures, Gricean maxims, relevance, obligatory irrelevance, exhaustivity, modified numerals, universal density of measurement

\section{Introduction}

Relevance considerations determine, in part, what inferences an utterance by a speaker can give rise to. Specifically, they delimit the range of scalar inferences (SIs) and (speaker-oriented) ignorance inferences (IIs) of assertions. The outcome of such considerations, i.e. the set of relevant propositions, is subject to certain constraints called closure conditions. Typically, these conditions reflect that the set of relevant propositions is conceived of as an exhaustive set of possible answers to a question under discussion (QUD). For instance, if, as we assume, QUDs partition the logical space (Groenendijk \& Stokhof 1984), then the set of relevant propositions must be closed under conjunction and negation (if $\phi$ and $\psi$ are relevant propositions, then so are $\phi \wedge \psi$ as well as $\neg \phi$ and $\neg \psi$; Lewis 1988; von Fintel \& Heim 1997). ${ }^{1}$ However,

* For helpful discussions, we thank Emmanuel Chemla, Liz Coppock, Luka Crnič, Danny Fox, Bernhard Schwarz, and Benjamin Spector, as well as the audiences of SALT 30, the workshop 'The Meaning of Numerals' at ZAS, the colloquium series at the University of Amsterdam, and the LINGUAE seminar series at the École Normale Supérieure.

1 The reason is that partitions are closed under intersection and complementation of their subsets: if $Q$ is a partition of $\mathscr{W}$, then for all $A, B \subseteq Q, A \cap B \subseteq Q$ and $\bar{A}, \bar{B} \subseteq Q$, where (nonempty proper) subsets of $Q$ represent possible complete or partial answers to $Q$.

C2020 Buccola and Haida 
How obligatory irrelevance, symmetric alternatives, and dense scales conspire

Fox (2016) proposes a closure condition of a different kind, viz. closure of relevance under (speaker) belief, stated in (1).

\section{Closure of relevance under belief}

If $\phi$ is relevant, then it's also relevant whether the speaker believes $\phi$.

Obviously, this condition cannot be justified by reference to formal properties of QUDs. Rather, its justification lies in the empirical observation that "silence is uncooperative", i.e. that ignorance about a subject matter doesn't warrant remaining silent when asked about that matter; rather, ignorance must be expressed, arguably because it's relevant (see Fox 2016; Buccola \& Haida 2019 for discussion). As may be easily seen, the condition in (1) interferes, possibly with major consequences, with the Maxim of Quantity (according to which, the speaker should utter a sentence that entails every relevant proposition that the speaker believes to be true): both state a condition with reference to what is relevant and to what the speaker believes. Indeed, adopting (1) entails accepting a quite radical view of how the Gricean conversational maxims interact with linguistic meanings. Specifically, the Maxim of Quantity no longer enriches linguistic meanings (Fox 2016); rather, it commits speakers to make their epistemic state about every relevant proposition explicit (Buccola \& Haida 2019). What this means, in more detail, is that SIs and IIs can only be derived in grammar, often via covert operators, and that speakers are bound to obey the condition in (2), where $K$ is the belief operator of S5 (Hintikka 1962) and $I \phi$ abbreviates $\neg K \phi \wedge \neg K \neg \phi$, i.e. ignorance about $\phi .^{2}$

\section{Strong Epistemic Transparency (SET) - a consequence of (1)}

An utterance $S$ must entail the speaker's epistemic state about every relevant proposition $\phi$. Epistemic states about $\phi$ are: (i) $K \phi$, (ii) $K \neg \phi$, and (iii) $I \phi$.

Note that, according to (2), $\neg K \phi$ on its own is not an epistemic state. ${ }^{3,4}$ This will turn out to be crucial for the analysis presented in this article. This article is about the contrast in IIs exhibited by non-strict comparative expressions like at least vs. strict ones like more than (hence the class A/B distinction of Nouwen 2010). Specifically, we argue that SET explains why numerals modified by more than cannot, on their own, induce IIs, while numerals modified by at least can.

2 Buccola \& Haida (2019) call this condition simply Epistemic Transparency. Thus, it is named identically to a weaker counterpart in an earlier work, viz. Meyer 2013. We realize that this is unfortunate and henceforth distinguish the two conditions nominally, even though the weaker version plays no role in this article.

3 The reason is that $\neg K \phi$ is compatible with both $K \neg \phi$ and $\neg K \neg \phi$. Thus, $\neg K \phi$ doesn't entail the speaker's epistemic state about $\neg \phi$, which is a relevant proposition given that $\phi$ is relevant and that relevance is closed under negation.

4 This is why SET is a stronger condition than its counterpart in Meyer 2013. 
Our article is structured as follows. In $\S 2$, we discuss empirical observations that establish the above-noted contrast between at least and more than for a special case, viz. numerals that are mapped onto an intuitively dense scale. $\S 3$ shows how SET explains this contrast. $\S 4$ refines the empirical picture we started out with and argues that recently discovered empirical phenomena provide additional support for our analysis. $\S 5$ extends the proposed analysis to intuitively non-dense scales. In $\S 6$, we discuss the observation that more than does not block SIs with round numerals and how this fact might be integrated with our analysis.

\section{Empirical background}

In this section, we consider numerals that, by their occurrence context, are mapped onto the weight scale. We thereby establish that the contrast in IIs exhibited by at least and more than, discovered by Geurts \& Nouwen (2007) with respect to intuitively non-dense scales, also holds for intuitively dense scales. The motivation for considering intuitively dense scales first is that SET makes immediate predictions for these (see §3), while an amendment is required for the others (see §5). The contrast of interest is exemplified by the sentences in (3) and (4). Sentence (3) implies that the speaker is ignorant about whether Bella weighs exactly $8 \mathrm{~kg}$ or more than $8 \mathrm{~kg}$ (see also Büring 2008; Nouwen 2010, 2015; Schwarz 2016), while (4) doesn't imply that the speaker is ignorant about whether Bella weighs exactly $9 \mathrm{~kg}$ or more than $9 \mathrm{~kg}$ (or about any other proposition).
Bella weighs at least $8 \mathrm{~kg}$.
(4) Bella weighs more than $8 \mathrm{~kg}$.

We will use the term unenriched meaning to refer to the meaning of a sentence in disregard of any and all SIs or IIs that it may be able to convey. The unenriched meaning of (3) and (4) is given in (5) and (6), respectively.
The unenriched meaning of (3) [ $\lambda w$. Bella's weight in $w \geq 8 \mathrm{~kg}$ ]
The unenriched meaning of (4)
[ $\lambda w$. Bella's weight in $w>8 \mathrm{~kg}$ ]

These propositions are abbreviated as given in (7) and (8), respectively, and likewise for similar propositions.

(7) $\quad[\geq 8]:=(5)$

Thus, we can characterize the contrast between (3) and (4) in the following way:

$$
\text { (3) } \rightsquigarrow I[>8], \quad(3) \rightsquigarrow I[=8]
$$
(4) $\not \rightarrow I[>9]$,
(4) $\not \rightarrow I[=9]$

We'll present a more nuanced discussion of these empirical claims in $\$ 4$. In the meantime, we note, building on an argument in Nouwen 2010, that the contrast 
How obligatory irrelevance, symmetric alternatives, and dense scales conspire

in IIs between at least vs. more than helps explain the intuitive contrast between sentences like??This $10 \mathrm{~kg}$ weight weighs at least $8 \mathrm{~kg}$ vs. This $10 \mathrm{~kg}$ weight weighs more than $8 \mathrm{~kg}$ : the former is odd because it obligatorily implies ignorance about how much the $10 \mathrm{~kg}$ weight weighs (while at the same time presupposing that it weighs exactly $10 \mathrm{~kg}$ ), whereas the latter is implies no such ignorance (and, at worst, is merely non-informative).

\section{Our explanation in a nutshell}

We now show that SET predicts that (4) cannot induce IIs while (3) can. ${ }^{5}$ To follow our argument, it's helpful to keep in mind that according to the assumptions we adopted in $\S 1$, a speaker is ignorant about a proposition $\phi, I \phi$, iff she is not certain that $\phi, \neg K \phi$, but considers $\phi$ possible, $\neg K \neg \phi$. As already mentioned, the pragmatic-semantic theory we explore in this article requires SIs and IIs to be derived in grammar. In the absence of overt expressions like only and I'm (un)certain that, we assume that SIs and IIs are invoked by syntactic occurrences of covert operators, specifically the exhaustification operator exh of Fox 2007 and the belief operator $K$ of Meyer 2013. With respect to the latter, we assume that it denotes (a Ty2 variant of) the belief operator of S5 (designated by the same symbol).

First, we turn to (3). Let's assume a context in which $[\geq 8],[=8]$, and $[>8]$ are all relevant propositions. How can a speaker convey in such a context (that she is certain about) $[\geq 8]$ while obeying SET with respect to the other two propositions? We claim that a speaker can do so by uttering the structure in (11).

$$
\left[{ }_{\gamma} \operatorname{exh}_{D}\left[{ }_{\beta} \mathrm{K}[\alpha \text { Bella weighs at least } 8 \mathrm{~kg}]\right]\right]
$$

In (11), a constituent $\alpha$, which denotes the unenriched meaning of (3), i.e. [ $\geq 8$, forms a constituent $\beta$ with an occurrence of the $K$ operator, which itself forms a constituent $\gamma$ with an occurrence of the exh operator. For the domain of exh, i.e. the set $D$, we assume that it contains $K[\geq 8], K[>8]$, and $K[=8]$ and no other proposition. ${ }^{6}$ To prove our claim, we note that $\beta$ denotes $K[\geq 8]$. By the semantics of exh, $\gamma$ denotes the conjunction of the denotation of $\beta$ with the negation of all members of $D$ whose negation is consistent with the denotation of $\beta$ and bears no logical implications for the other members of $D$. Hence, $\gamma$ denotes $K[\geq 8] \wedge$

5 We believe that it's for independent reasons that, outside of echoic contexts (see §4), at least always comes with IIs. See Spector 2015 for an account that we hope might be integrated with ours.

6 The domain of exh is restricted by two sets, viz. the set of relevant alternatives and the set of formal alternatives (Fox \& Katzir 2011). We hence assume that $K[>8]$ and $K[=8]$ are denotations of formal alternatives of the constituent $\beta$ in (11). We will discuss this assumption in $\S 6$. We can ignore all other alternatives because they're either entailed by $\beta$ or they turn out to be 'obligatorily irrelevant' (Buccola \& Haida 2019), as the discussion in this section will allow the reader to infer. 
$\neg K[>8] \wedge \neg K[=8]$, since $K[\geq 8] \wedge \neg K[>8]$ is consistent and logically independent of $K[=8]$, and likewise $K[\geq 8] \wedge \neg K[=8]$ is consistent and logically independent of $K[>8]$ (while $K[\geq 8] \wedge \neg K[\geq 8]$ is inconsistent). See (12) for a summary.

\section{The denotations of the labeled constituents in (11)}

$$
\begin{aligned}
\llbracket \alpha \rrbracket^{g}= & {[\geq 8] } \\
\llbracket \beta \rrbracket^{g}= & K[\geq 8] \\
\llbracket \gamma \rrbracket^{g}= & K[\geq 8] \wedge \neg K[>8] \wedge \neg K[=8] \\
& \text { for } g(D)=\{K[\geq 8], K[>8], K[=8]\}
\end{aligned}
$$

What remains to be shown is that the denotation of $\gamma$ logically entails $I[>8]$ and $I[=8]$. That this is indeed the case follows from the logical facts noted in (13).

$$
K[\geq 8] \wedge\left\{\begin{array}{lll}
\neg K[>8] & \Rightarrow \neg K \neg[=8] \\
\neg K[=8] & \Rightarrow \neg K \neg[>8]
\end{array}\right.
$$

Overall, in using (11), the speaker expresses $K[\geq 8] \wedge I[=8] \wedge I[>8]$. Thus, she can convey $[\geq 8]$ while being transparent about $[=8]$ and $[>8]$, namely by expressing her ignorance about the latter.

Turning to (4), we start out by making parallel assumptions as with the foregoing example. Specifically, we assume that the propositions $[>8],[>9]$, and $[=9]$ are all relevant, and that the speaker utters the structure in (14), where $D$ is the set $\{K[>8], K[>9], K[=9]\}$.

$$
\left[\gamma \operatorname{exh}_{D}[\beta \mathrm{K}[\alpha \text { Bella weighs more than } 8 \mathrm{~kg}]]\right]
$$

We claim that by uttering (14) the speaker fails to obey SET with respect to $[>9]$ and $[=9]$. Here is the reason why. The labeled constituents in (14) have analogous denotations to their counterparts in (11), see (15).

\section{The denotations of the labeled constituents in (14)}

$$
\begin{aligned}
\llbracket \alpha \rrbracket^{g}= & {[>8] } \\
\llbracket \beta \rrbracket^{g}= & K[>8] \\
\llbracket \gamma \rrbracket^{g}= & K[>8] \wedge \neg K[>9] \wedge \neg K[=9] \\
& \text { for } g(D)=\{K[>8], K[>9], K[=9]\}
\end{aligned}
$$

However, unlike before, the denotation of $\gamma$ does not support the possibility inferences $\neg K \neg[=9]$ and $\neg K \neg[>9]$ to complement the uncertainty entailments $\neg K[>9]$ and $\neg K[=9]$ to yield the ignorance inferences $I[>9]$ and $I[=9]$, see (16).

$$
K[>8] \wedge\left\{\begin{array}{lll}
\neg K[>9] & \nRightarrow & \neg K \neg[=9] \\
\neg K[=9] & \nRightarrow & \neg K \neg[>9]
\end{array}\right.
$$


How obligatory irrelevance, symmetric alternatives, and dense scales conspire

The reason is that $K[>8] \wedge \neg K[=9] \wedge \neg K[>9]$ is compatible with e.g. $K[=8.5]$, which entails $K \neg[=9]$ and $K \neg[>9]$. This means that a speaker cannot convey [>8] while being epistemically transparent about $[>9]$ and $[=9] . .^{7}$ The same argument can be replicated for any pair of alternatives $[>n]$ and $[=n]$ with $n>8$ given that the weight scale is dense. What this means is that $[>8]$ can only be conveyed in contexts in which all of these stronger alternatives are irrelevant. This is the property called obligatory irrelevance by Buccola \& Haida (2019), and we submit that this explains why more than cannot induce IIs.

\section{Empirical support}

Two recent experimental studies (Westera \& Brasoveanu 2014; Cremers, Coppock, Dotlačil \& Roelofsen 2017) found that the contrast in IIs between at least and more than is to a certain extent context dependent. Specifically, explicit context questions seem to modulate the propensity of hearers to draw IIs from sentences like (3) and (4). We argue that these findings support the SET approach. We begin with presenting (3) and (4) in the context of indirect polar questions, which brings out the contrast of interest in an unobstructed way (Cremers et al. 2017). ${ }^{8}$

\{Choppi is food for dogs that weigh less than $8 \mathrm{~kg}\}$

Q: Is Choppi suitable for Bella?

A: Bella weighs at least $8 \mathrm{~kg}$. (So the answer is no.)
(18) \{Choppi is food for dogs that weigh at most $8 \mathrm{~kg}$ \}

Q: Is Choppi suitable for Bella?

A: Bella weighs more than $8 \mathrm{~kg}$. (So the answer is no.)

In (17) and (18), the text in curly brackets provides information about the common knowledge of the interlocutors. On the background of these pieces of information, the question in (17/18-Q) asks different polar questions about Bella's weight: in the case of (17), it provides the answer set $\{[<8],[\geq 8]\}$, and in the case of (18) the answer set $\{[\leq 8],[>8]\}$. The two questions differ so that either one is settled by the unenriched meaning of its respective answer: the unenriched meaning of (17-A), i.e. [ $\geq 8]$, is compatible with just one member of the former answer set, and the unenriched meaning of (18-A), i.e. [>8], with just one member of the latter one. Nevertheless, we observe, supported by quantitative data in Cremers et al. 2017, that the two answers differ in what they convey about the speaker's beliefs: (17-A) implies ignorance about $[>8]$ and about $[=8]$, while no ignorance

7 Recall that according to SET, $\neg K \phi$ (without $\neg K \neg \phi$ ) is not an epistemic state (see note 3).

8 Both Westera \& Brasoveanu 2014 and Cremers et al. 2017 are concerned with sentences involving intuitively non-dense scales. Based on our own judgments, we believe that the variation reported in these studies is independent of this feature. Therefore, we continue using examples with intuitively dense scales. 
is implied by (18-A). As the discussion in $\S 3$ shows, the SET account predicts two crucial components of this contrast: it predicts that (17-A) can be associated with truth conditions that have the observed IIs as entailments, while (18-A) cannot be associated with ignorance-entailing truth conditions.

Two points should be noted about the contexts just considered. First, the answer set specified in (17), i.e. $\{[<8],[\geq 8]\}$, cannot be equated with the QUD from which the set of relevant propositions is derived: obviously, $[>8]$ and $[=8]$ are not (disjunctions of) members of this answer set; hence, if we equated the QUD with this answer set (or rather its closure under belief), we would falsely predict no IIs to arise from (17-A). Second, it's crucial to express the polar questions of interest in an indirect way, as will become clear immediately.

Quantitative data presented by Westera \& Brasoveanu (2014) suggest that context questions can obviate IIs of at least. Specifically, in the context of the polar question in (19-Q), the use of (3)/(19-A) does not imply speaker ignorance. In particular, (19-Q) specifies the same polar question as the context question in (17) but does so in a direct way.

A: Bella weighs at least $8 \mathrm{~kg}$.

The obviation of IIs in echoic contexts is predicted by the theory our account is based on. As Buccola \& Haida (2019) show, the assumption that relevance is closed under speaker belief (from which SET follows) forces the adoption of exh, in order for speakers to be able to obey SET. Crucially, a proposition can only be in the domain of exh if it's a focus alternative of its prejacent (see §6.2.2 and Katzir 2007; Fox \& Katzir 2011). In the context of (19), every constituent of (3) is given and hence unfocused (with the possible exception of constituents representing the assertive force of this sentence). Specifically, the modified numeral at least 8 is unfocused. Consequently, $K[=8]$ and $K[>8]$ aren't focus alternatives of $K[\geq 8]$ and hence not in the domain of exh. Therefore, no IIs can be derived.

We now turn to contexts that evoke a reponse by means of a degree question, specifically the question in (20-Q). First, we consider the case of a speaker responding with the more than sentence in (4), see (20-A).

$$
\text { Q: How much does Bella weigh? }
$$

A: Bella weighs more than $8 \mathrm{~kg}$.

Westera \& Brasoveanu (2014) and Cremers et al. (2017) report that experimental subjects draw an inference of speaker ignorance when being exposed to questionanswer sequences such as (20). We contend that this inference is not grammatically induced in the way explained for at least sentences. Rather, it's a pragmatic inference of sorts which arises because of the following properties of (20). The degree question in (20-Q) denotes the answer set $\{[=d]: d \in \mathbb{Q}\}$ (including e.g. the proposition that Bella weighs exactly $8.5 \mathrm{~kg}$; see Spector 2018). Consequently, (20-A) provides an 
How obligatory irrelevance, symmetric alternatives, and dense scales conspire

answer to (20-Q), however, only a partial one: it eliminates the subset $\{[=d]: d \in$ $\mathbb{Q} \wedge d \leq 8\}$ from the answer set, but it's compatible with all of its other members. Moreover, as shown in $\S 3,(20-\mathrm{A})$ renders all stronger alternatives $[>n]$ and $[=n]$ with $n>8$ obligatorily irrelevant because a speaker cannot convey [ $>8]$ and be epistemically transparent about these alternatives. This means that (20-A) as a response to (20-Q) renders every compatible member of the answer set of (20-Q) irrelevant. That is, it dodges (a big chunk of) the question. We believe that this is why one might draw the reported inference of speaker ignorance, since one reason for dodging a question is (to convey) ignorance. To see that there is a difference between the inferences induced by at least vs. more than in the context of a degree question, note the contrast between $A_{1}$ and $A_{2}$ as a response to $Q$ in (21): $A_{1}$ is odd since it entails ignorance about one's own age (cf. Nouwen 2010); $\mathrm{A}_{2}$, in contrast, isn't odd since it doesn't entail ignorance, and since there are other reasons for dodging a question (like not wanting to disclose one's actual age).

Q: How old are you? $\quad \mathrm{A}_{1}$ : \#I'm at least $40 . \quad \mathrm{A}_{1}:$ I'm more than 40.

The last case study of this section is concerned with at least sentences in the context of a degree question, see below.

Q: How much does Bella weigh?

A: Bella weighs at least $8 \mathrm{~kg}$.

Cremers et al. (2017) report that question-answer discourses like (22) evoke different behavior from experimental subjects than question-answer discourses like (17): subjects draw a stronger inference of speaker ignorance from (22). Again, we believe that the key in explaining this fact is the observation that (22-A) gives an incomplete answer to (22-Q) and that it renders all but one of the compatible members of the answer set of (22-Q) obligatorily irrelevant. More precisely, (22-A) eliminates the subset $\{[=d]: d \in \mathbb{Q} \wedge d<8\}$ from the answer set and is compatible with all of its other members. Furthermore, (22-A) renders all stronger alternatives except for $[>8]$ and $[=8]$ obligatorily irrelevant. Thus, it renders every compatible proposition in the answer set of (22-Q) irrelevant, except for the proposition $[=8]$, which is a proposition that (22-A) entails ignorance about. Again, this means that the response in (22) is dodging the question. Therefore, a subject encountering the question-answer discourse in (22) has two reasons to infer that the answerer is ignorant: first, because of the ignorance entailments of (22-A), and second, because the answerer is dodging the question. We submit that this explains the observation of Cremers et al. (2017). Thus, we agree with these authors that there are 'two routes to ignorance', and that cases involving both routes induce an additive effect. However, we submit that the two routes are (i) grammatically induced IIs and (ii) pragmatic inferences that give an interpretation to question-dodging behavior. 
Buccola and Haida

\section{Adapting the SET account to non-dense scales}

The argument presented in $\S 3$ relies on the intuitive assumption that the weight scale is dense. The counting scale, in contrast, is intuitively discrete. Still, we observe the same contrast between (23) and (24) as we do between (3) and (4): only (23) implies ignorance.

(23) Ann owns at least two dogs.

(24) Ann owns more than two dogs.

Without further assumptions, however, (24) is wrongly predicted, under our account, to be able to induce IIs. To see this, consider (25), which, as we did in $\$ 3$, we hypothesize to be a parse of (24) with $D$ being the set $\{K[>2], K[>3], K[=3]\}$.

$$
\left[\gamma \operatorname{exh}_{D}\left[{ }_{\beta} \mathrm{K}[\alpha \text { Ann owns more than } 2 \text { dogs }]\right]\right]
$$

The denotation we derive for (24) is provided in (26), where $[>2]$ is the proposition $[\lambda w$. the number of dogs owned by Ann in $w>2]$ (and likewise for $[>3]$ and $[=3]$ ):

\section{The denotations of the labeled constituents in (25)}

$$
\begin{aligned}
\llbracket \alpha \rrbracket^{g}= & {[>2] } \\
\llbracket \beta \rrbracket^{g=} & K[>2] \\
\llbracket \gamma \rrbracket^{g=} & K[>2] \wedge \neg K[>3] \wedge \neg K[=3] \\
& \text { for } g(D)=\{K[>2], K[>3], K[=3]\}
\end{aligned}
$$

On the intuitive assumption that (25) involves the counting scale, the logical facts in (27) are supported. As a consequence, the denotation of $\gamma$ logically entails $I[>3]$ and $I[=3]$, so that we incorrectly predict that (24) can induce IIs.

$$
K[>2] \wedge\left\{\begin{array}{lll}
\neg K[>3] & \Rightarrow & \neg K \neg[=3] \\
\neg K[=3] & \Rightarrow & \neg K \neg[>3]
\end{array}\right.
$$

The situation we face here for (4) and (24) with respect to IIs mirrors what Fox \& Hackl (2006) observe with respect to SIs: there is an explanation for why (4) cannot be used to convey that Bella weighs exactly $9 \mathrm{~kg}$ (or exactly $8.5 \mathrm{~kg}, 8.25 \mathrm{~kg}$, ...), which relies on the weight scale being dense. ${ }^{9}$ However, despite the intuitive difference in granularity between the weight scale and the counting scale, (24) cannot be used to convey that Ann owns exactly three dogs either. Fox \& Hackl (2006) solve this puzzle by hypothesizing that measurement scales needed for natural language semantics are always dense. This so-called universal density of measurement (UDM)

9 The explanation is that there is no smallest degree greater than 8: for every degree $d>8$, there is a degree greater than 8 but smaller than $d$ (since the weight scale is dense). Thus, $[>8]$ has no weakest stronger alternative $[>d]$. However, the exclusion of all stronger alternatives contradicts $[>8]$. As a consequence, exhaustification cannot apply (relative to the dense alternative set). 
How obligatory irrelevance, symmetric alternatives, and dense scales conspire

hypothesis solves our puzzle in a similar fashion: with the UDM in place, the logical entailments in (27) no longer hold, and so the denotation in (26) no longer supports the IIs $I[>3]$ and $I[=3]$ as logical entailments. In fact, the alternatives $[>3]$, [=3], and all $[>n]$ and $[=n]$ for $n>2$ are obligatorily irrelevant, in complete parallel to what we demonstrated for (4) in $\S 3$, and that's why (24) cannot induce IIs.

This is the desired result. However, we also need to take care of the following observation, which has the potential to obviate the desired result: on the intuitive assumption that the proposition $[>2]$ is contextually equivalent to the proposition $[\geq 3$, we derive the contextual entailments in (28), where $c$ is a context such that $[>2] \equiv_{c}[\geq 3]$.

$$
K[>2] \wedge\left\{\begin{array}{lll}
\neg K[>3] & \Rightarrow_{c} & \neg K \neg[=3] \\
\neg K[=3] & \Rightarrow_{c} & \neg K \neg[>3]
\end{array}\right.
$$

Therefore, if we want to generalize the SET approach from sentences involving intuitively dense scales to sentences involving intuitively non-dense scales, we must assume, in addition to the UDM, that SET is a condition on the logical entailments of an utterance. This is stated in (29) as a hypothesis.

\section{Hypothesis about SET (to be tentatively rejected)}

The entailment notion that figures in the formulation of SET in (2) is the notion of logical entailment.

As a consequence, (29) implies that the entailment notion that figures in the formulation of the Maxim of Quantity must also be logical entailment. The reason is that SET is a consequence of the interaction between the Maxim of Quantity and the assumption that relevance is closed under belief. Buccola \& Haida (2019: §6.4) provide methodological motivation and empirical evidence for the assumption that the relevant notion is logical entailment. However, this assumption seems to be at odds with the Maxim of Quantity being a conversational principle, which should rather be involved with contextual entailment. In the next section, we explore how to reject this assumption while also improving the empirical coverage of our approach.

\section{An alternative account}

\subsection{Conceptual and empirical issues to be addressed}

As we've just seen, generalizing the SET account of the contrast in IIs between at least and more than requires the assumption that the Maxim of Quantity commits speakers to utter sentences that logically entail every contextually relevant proposition they believe to be true. However, for conceptual reasons it's much more plausible that the Maxim of Quantity is concerned with contextual entailment. 
Therefore, we now investigate an alternative to adopting (29). In addition, our investigation aims to broaden the empirical coverage of the aspired analysis. The current analysis accounts for the IIs in (30) and the absence of IIs and SIs in (31). ${ }^{10}$

at least induces IIs (and not SIs)

$$
\begin{aligned}
& \text { (3) } \rightsquigarrow I[>8] \text {, } \\
& \text { (23) } \rightsquigarrow I[>2] \text {, } \\
& \text { (3) } \rightsquigarrow I[=8] \\
& \text { (23) } \rightsquigarrow I[=2]
\end{aligned}
$$

more than does not induce IIs/SIs

$$
\begin{aligned}
& \text { (4) } \leftrightarrow \rightarrow I[>9] \text {, } \\
& \text { (4) } \not \rightarrow I[=9] \\
& \text { (24) } \not \rightarrow I[>3] \text {, } \\
& \text { (24) } \leftrightarrow \rightarrow[=3] \\
& \text { (4) } \nLeftarrow \rightarrow K[=9] \text {, } \\
& \text { (24) } \not \rightarrow K[=3]
\end{aligned}
$$

However, it does not predict another set of facts reported in the recent literature, viz. the availability of SIs with (3), see (32), and the sentences in (33), where [>200] abbreviates the proposition $[\lambda w$. the number of people that attended in $w>200]$ and likewise for $[=200],[>100]$, and $[\geq 100]^{11}$

(3) $\rightsquigarrow \neg[>9] \wedge \neg[=9]$

(Enguehard 2018)

\{More than $\mid$ At least $\} 100$ people attended. $\rightsquigarrow \neg[>200] \wedge \neg[=200]$

(cf. Cummins et al. 2012)

Thus, in what follows we not only abandon (29) but also aim to predict SIs of modified round numerals. ${ }^{12}$ The alternative analysis we propose will be compatible with SET but it will explain the facts in (30) and (31) independently from it. Nevertheless, we continue to explore the consequences of closure of relevance under belief even though, as just pointed out, this condition will no longer play a crucial part in explaining the empirical observations reported above.

\subsection{Restrictions on exhaustification domains}

\subsubsection{Our goals}

Here, we set the stage for a later assumption, namely that exhaustification must apply below the $K$ operator. The desired effect of this assumption is to preempt the derivation of unattested IIs (caused, without preemption, by subsequent exhaustification above $K$ ). Preemption of IIs is achieved by imposing restrictions on exhaustification domains. To enable a more specific discussion, we adopt the following standard definition of exh (Fox 2007; Magri 2009): ${ }^{13}$

10 Recall that the absence of SIs follows from the UDM, which we adopted in $\S 5$.

11 Cummins et al. (2012) only discuss the SI $\neg[>200]$ for sentences like the more than variant of (33). They remain silent about the exclusion of $[=200]$ with more than 100 and don't make specific claims about the SIs of at least 100. However, our empirical claims in (33) are consistent with their quantitative data.

12 The numeral 8 in (3) is a round numeral in that it denotes an integer on a scale of rational numbers.

13 Usually, we assume, for expository reasons, that the domain of exh is assigned to a syntactic variable that comes with syntactic occurrences of exh. The rendering in (34) is better suited to our current 
How obligatory irrelevance, symmetric alternatives, and dense scales conspire

$$
\begin{aligned}
& \text { The definition of exh } \\
& \llbracket \operatorname{exh} S \rrbracket^{g}=[\lambda w \cdot p(w)=1 \wedge \forall q \in \operatorname{IE}(A, p): q(w)=0] \text {, where: } \\
& p=\llbracket S \rrbracket^{g} \\
& A=\left\{\llbracket S^{\prime} \rrbracket^{g}: S^{\prime} \in \operatorname{Alt}(S)\right\} \cap R \\
& \operatorname{IE}(A, p)=\bigcap\{E: E \text { is a maximal subset of } A \text { such that } p \wedge \neg \bigvee E \not \equiv \perp\}
\end{aligned}
$$

Our discussion concerns the following two components of this definition, where $S$ is the structure undergoing exhaustification: (i) the set $\operatorname{Alt}(S)$ of formal alternatives of $S$ and (ii) the set $R$ of relevant propositions. The set $A$ of relevant propositions denoted by formal alternatives of $S$ is what we call 'exhaustification domain' or 'domain of exh' in previous sections and subsequently.

With regard to (i), Table 1 shows how the structures of interest are to be associated with alternative sets, where the associations vary with whatever is the intuitively adequate scale for interpreting the structure in context. In this table, we characterize structures and alternative-set members by their denotations. ${ }^{14}$ We do this for brevity and because the assignment-function parameter $g$ of the interpretation function will serve to determine the granularity level of the alternative sets.

With regard to (ii), we need to restrict the extensions of $R$ in such a way that certain subsets of $\left\{\llbracket S^{\prime} \rrbracket^{g}: S^{\prime} \in \operatorname{Alt}(S)\right\}$ are preserved if they emerge. These restrictions will impose limits on which members of triplets like $\{[>2],[>3],[=3]\}$ can be disregarded. Specifically, they rule out $R=\{[>2],[>3]\}$ so that $[>2]$ in the fourth row of Table 1 cannot be exhaustified relative to the domain $\bigcup_{n \in \mathbb{N}}\{[>n],[=n]\} \cap$ $\{[>2],[>3]\}$, since this would yield the SI $\neg[>3]$ and hence the unwanted enriched meaning $[=3]$ (given the UDM and the contextual equivalence of $[>2]$ and $[\geq 3]$ ).

\subsubsection{Providing the generative capacity to build the desired $\operatorname{Alt}(S)$ values}

We adopt the theory of formal alternatives proposed in Katzir 2007; Fox \& Katzir 2011. In this theory, the set of formal alternatives of a structure $S$, i.e. $\operatorname{Alt}(S)$, is defined as in (35), where $\operatorname{Alt}_{\text {Rooth }}(S)$ is the set of focus alternatives of $S$ (Rooth 1985, as given in Fox \& Katzir 2011: 93) and $\operatorname{Alt}_{\text {Katzir }}(S)$ contains all and only those structures $S^{\prime}$ that can be derived from $S$ by replacing constituents of $S$ with items of the same or lower structural complexity (see the cited references for details).

purposes. With (34), we are simplifying matters somewhat since $\operatorname{Alt}(S)$ is to a certain degree context dependent (Katzir 2007). We believe that this simplification doesn't impede the discussion.

14 We don't want to suggest that the alternative-building mechansim derives the desired associations without reference to how the propositions in the first column of Table 1 are linguistically expressed. For example, the parse of the sentence Bella weighs no less than $8 \mathrm{~kg}$ contains a structure denoting $[\geq 8]$ (Nouwen 2008). However, because of restrictions limiting the generative capacity of the alternative-building mechanism, this structure cannot be associated with the alternative set in the first row of Table 1 (Haida 2019). 


\begin{tabular}{lcl}
\hline$\llbracket S \rrbracket^{g}$ & $\begin{array}{c}\text { Intuitive scale suggested by } S \\
\text { and/or its context }\end{array}$ & $\left\{\llbracket S^{\prime} \rrbracket^{g}: S^{\prime} \in \operatorname{Alt}(S)\right\}$ \\
\hline$[\geq 8]$ & dense & $\bigcup_{d \in \mathbb{Q}^{+}}\{[\geq d],[>d],[=d]\}$ \\
\hline$[>8]$ & & $\bigcup_{d \in \mathbb{Q}^{+}}\{[>d],[=d]\}$ \\
\hline$[\geq 2]$ & non-dense with steps of 1 & $\bigcup_{n \in \mathbb{N}}\{[\geq n],[>n],[=n]\}$ \\
\hline$[>2]$ & & $\bigcup_{n \in \mathbb{N}}\{[>n],[=n]\}$ \\
\hline$[\geq 100]$ & non-dense with steps of 100 & $\bigcup_{c \in\{n \cdot 100: n \in \mathbb{N}\}}\{[\geq c],[>c],[=c]\}$ \\
\hline$[>100]$ & & $\bigcup_{c \in\{n \cdot 100: n \in \mathbb{N}\}}\{[>c],[=c]\}$
\end{tabular}

Table 1 Desired values of $\operatorname{Alt}(S)$ for various (denotations of) $S$

$$
\operatorname{Alt}(S)=\operatorname{Alt}_{\text {Rooth }}(S) \cap \operatorname{Alt}_{\text {Katzir }}(S)
$$

In general terms, the Katzir alternatives of the structures under consideration are derived by (I) replacing numeral modifiers with other numeral modifiers and/or (II) replacing numerals with other numerals (cf. Schwarz 2016). It's beyond the scope of this article to defend the first assumption (or assumptions to its effect). Therefore, we merely acknowledge our acceptance of the stipulations in (36).

a. The numeral modifiers at least, more than, and exactly are constituents of expressions they occur in.

b. i. More than is no more complex than at least.

ii. Exactly is no more complex than more than.

By (36), the alternative-building mechanism can derive e.g. ( $\alpha$ ) Bella weighs more than $8 \mathrm{~kg}$ and $(\beta)$ Bella weighs exactly $8 \mathrm{~kg}$ from $(\gamma)$ Bella weighs at least $8 \mathrm{~kg}$, and $(\beta)$ from $(\alpha)$.

Assumption (II), we spell out in the following way. We assume that numerals denote degrees (of the atomic semantic type $d$; cf. Landman 2004) and that they can be moved at LF (cf. Kennedy 2015). Specifically, if a numeral is c-commanded by an exh operator, it can be moved to a position c-commanding that operator. ${ }^{15}$ Thus, we assume, for instance, that (37) is a possible LF structure (and likewise for structures without a numeral modifier or with a different one).

15 Presumably, movement of this type is restricted by locality constraints. We have to leave further explorations to future research. 
How obligatory irrelevance, symmetric alternatives, and dense scales conspire

$$
\text { [ } \left.\left.{ }_{\beta} \text { two }\left[\lambda_{1} \text { [exh }\left[\alpha \text { Ann owns more than } t_{1} \text { dogs }\right]\right]\right]\right]
$$

Above, $t_{1}$ is a syntactic variable of the same type as its antecedent, i.e. a variable of type $d$. Because of the unbounded nature of language, there must be an infinite repository of syntactic variables of type $d$. For concreteness, let this repository be the set $T_{d}=\left\{t_{n}: n \in \mathbb{N}\right\}$. We assume that all of the variables in $T_{d}$ are equally complex (i.e. differences in the formal complexity of subscripted indices are notational artefacts). Consequently, $t_{1}$ in (37) can be replaced with any other syntactic variable in $T_{d}$. Together with (36b-ii), we thus derive the following set of Katzir alternatives of the complement $\alpha$ of the exh operator in (37):

$$
\begin{aligned}
\operatorname{Alt}_{\text {Katzir }}(\alpha)= & \left\{\left[\text { Ann owns more than } t_{n} \operatorname{dogs}\right]: n \in \mathbb{N}\right\} \\
& \cup\left\{\left[\text { Ann owns exactly } t_{n} \operatorname{dogs}\right]: n \in \mathbb{N}\right\}
\end{aligned}
$$

This means that the set of Katzir alternatives of $\alpha$ yields the proposition set in (39) under the interpretation function $\llbracket \cdot \rrbracket^{g}$.

$$
\left\{\llbracket \alpha^{\prime} \rrbracket^{g}: \alpha^{\prime} \in \operatorname{Alt}_{\text {Katzir }}(\alpha)\right\}=\bigcup_{d \in D}\{[>d],[=d]\}
$$

Above, the set of degrees $D$ is the image of the set of syntactic variables of type $d$ under $g$, i.e. $D=\left\{g(t): t \in T_{d}\right\}$. Thus, $D$ can be $\mathbb{Q}^{+}$(a dense scale), $\mathbb{N}$ (a non-dense scale with steps of 1), $\{n \cdot 100: n \in \mathbb{N}\}$ (a non-dense scale with steps of 100), or any other set of degrees, depending on the parameter $g$ of the interpretation function. Since, by standard assumptions, the argument-value associations of assignment functions are context dependent, we thus derive, at the level of Katzir alternatives, the context-dependent variation in Table 1 for structures containing more than and, mutatis mutandis, also for structures containing at least.

Let's assume, for illustration, that the domain of exh in (37) is the set $\bigcup_{d \in \mathbb{N}}\{[>$ $d],[=d]\}$. Then, the constituent $\beta$ has the following denotation, where the first equivalence holds if (and only if) the UDM is valid. ${ }^{16}$

$$
\begin{aligned}
\llbracket \beta \rrbracket^{g}=[\lambda w . & \llbracket \text { Ann owns more than } t_{1} \operatorname{dogs} \rrbracket^{g\left[t_{1} \mapsto 2\right]}(w)=1 \\
& \left.\wedge \forall q \in \operatorname{IE}\left(\bigcup_{d \in \mathbb{N}}\{[>d],[=d]\}, \llbracket \alpha \rrbracket^{g}\right): q(w)=0\right] \\
\equiv \lambda w \cdot & {\left[\left[\lambda w^{\prime} . \text { the number of dogs Ann owns in } w^{\prime}>2\right](w)=1\right.} \\
& \left.\wedge \forall q \in \bigcup_{d \in \mathbb{N} \backslash\{0,1,2\}}\{[>d],[=d]\}: q(w)=0\right] \\
\equiv[\lambda w . & \text { the number of dogs Ann owns in } w>2 \\
& \wedge \text { the number of dogs Ann owns in } w \ngtr 3 \\
& \wedge \text { the number of dogs Ann owns in } w \neq 3]
\end{aligned}
$$

Obviously, the proposition derived above is a contextual contradiction. As will become apparent shortly, this plays an important role in our preemption account.

16 This is because the condition of innocent excludability requires logical consistency, which is met because $[>2] \wedge \neg[>3] \wedge \neg[=3]$ is logically consistent given the UDM. 
Buccola and Haida

\subsubsection{Restrictions on $R$}

We've explored the consequences of the assumption that the set $R$ of relevant propositions is closed under conjunction, negation, and speaker belief, which we adopted in $\S 1$ and kept, although we changed course at the beginning of this section. Here we add another closure condition, viz. closure of $R$ under contextual equivalence (Magri 2009), given in (41):

(41) If $\phi \in R$ and $\psi$ is contextually equivalent to $\phi$, then $\psi \in R$.

In conjunction with the other closure conditions, (41) implies that $R$ contains all contextual equivalences of Boolean combinations of its members. For example, if $c$ is a context that supports $[>2] \equiv_{c}[\geq 3]$, then we derive the following consequences regarding $R$ :

$$
\begin{aligned}
{[>2],[>3] \in R } & \Rightarrow[>2] \wedge \neg[>3] \in R & & (\text { closure under } \wedge \text { and } \neg) \\
& \Rightarrow[=3] \in R & & \left(\text { closure under } \equiv_{c}\right)
\end{aligned}
$$

Thus, (41) guarantees that $R$ cannot 'break contextual symmetry': if $R$ contains $[>2]$ in a context $c$ that supports $[>2] \equiv_{c}[\geq 3]$ and hence the symmetric contextual entailments in (43), then $R$ contains either none or both of $[=3]$ and $[>3] .{ }^{17}$

$$
[>2] \wedge\left\{\begin{array}{lll}
\neg[>3] & \Rightarrow_{c} & {[=3]} \\
\neg[=3] & \Rightarrow_{c} & {[>3]}
\end{array}\right.
$$

This is a good result because broken contextual symmetries yield unwanted SIs, which we prevent with (41).

Note that we also derive that, in the same context, $R$ is a dense set: ${ }^{18}$

$$
[>2] \in R \Rightarrow[>d] \in R \text { for all } d \in \mathbb{Q} \text { with } 2<d<3 \quad \text { (closure under } \equiv_{c} \text { ) }
$$

The result in (44) is counterintuitive but unproblematic for our approach, since, as just shown, the alternative-building mechanism yields non-dense alternative sets in contexts that suggest a non-dense scale with steps of 1 (or other step sizes).

17 For triplets like $\{[\geq 8],[>8],[=8]\}$ and $\{K[\geq 8], K[>8], K[=8]\}$, symmetry breaking is prevented by closure of relevance under conjunction, negation, and belief alone since the symmetric entailments these triplets support are logical entailments and not just contextual entailments.

18 If the UDM is valid, the dense ordering of the rational interval $[2,3$ ) carries over to a dense logicalstrength ordering of $R$ : if $R$ satisfies the condition in (44), $R$ contains, for any two of its members $[>d],\left[>d^{\prime}\right]$ with $3>d>d^{\prime} \geq 2$, a third member $\left[>d^{\prime \prime}\right]$ with $d>d^{\prime \prime}>d^{\prime}$; because of the UDM, $[>d] \subset\left[>d^{\prime \prime}\right] \subset\left[>d^{\prime}\right]$, where ' $\subset$ ' is the asymmetric (logical) entailment relation. 
How obligatory irrelevance, symmetric alternatives, and dense scales conspire

\subsection{An LF hypothesis}

On the background of our proposals regarding $\operatorname{Alt}(S)$ and $R$, the main ingredients of our new analaysis are the UDM together with the structural hypothesis in (45).

\section{LF hypothesis for (assertive) sentences}

Every sentence has the following hierarchy of covert operators in its periphery: $\left[\operatorname{exh}_{D_{2}}\left[\mathrm{~K}\left[\operatorname{exh}_{D_{1}} \ldots\right]\right]\right]$.

In (45), $D_{2}$ is the set $\left\{K p: p \in D_{1}\right\} .{ }^{19}$ This means that if we start out, for example, with the hypothesis that $D_{1}=\{[>2],[>3],[=3]\}$ and find that, for some reason, $D_{1}$ must exclude $[>3]$ and [ $=3]$, we can further conclude that $D_{2}$ must exclude $K[>3]$ and $K[=3]$.

We start out exploring the consequences of (45) by considering the structure it imposes on (24), see the LF strcuture in (46). This structure also exhibits LF movement of the numeral two to a position above the lower exh operator.

$$
\left[\operatorname{exh}_{D_{2}}\left[\mathrm{~K}\left[\beta \text { two }\left[\lambda_{1}\left[\operatorname{exh}_{D_{1}}\left[\alpha \text { Ann owns more than } t_{1} \text { dogs }\right]\right]\right]\right]\right]\right]
$$

The foregoing discussion allows us to infer the value assignments in (47a) and assume the value assignments in (47b) to derive the value assignment in (47c).

$$
\begin{aligned}
& \text { a. } \llbracket \alpha \rrbracket^{g\left[t_{1} \mapsto 2\right]}=[>2] \\
& g\left(D_{1}\right)=\{\llbracket \alpha^{\prime} \rrbracket^{g}: \alpha^{\prime} \in \overbrace{\operatorname{Alt}_{\text {Katzir }}(\alpha)}^{A} \cap \overbrace{\operatorname{Alt}_{\text {Rooth }}(\alpha)}\} \cap R \\
& \left\{\llbracket \alpha^{\prime} \rrbracket^{g}: \alpha^{\prime} \in A_{1}\right\}=\bigcup_{n \in \mathbb{N}}\{[>n],[=n]\} \quad=: A_{3} \\
& \text { b. } A_{2} \supseteq A_{1}, R \supseteq\{[>2],[>3],[=3]\} \\
& \text { c. } g\left(D_{1}\right)=\{[>2],[>3],[=3]\} \cup\left(A_{3} \cap R\right)
\end{aligned}
$$

The propositions in $A_{3} \cap R$ and not in $\{[>2],[>3]$, [ $\left.=3]\right\}$ (i.e. denotations of Katzir alternatives such as $[>1]$ or $[=4]$ ) play no role for the outcome of exhaustifying $\llbracket \alpha \rrbracket^{g\left[t_{1} \mapsto 2\right]}$ relative to $g\left(D_{1}\right)$ and will hence be ignored. Thus, we can derive the following denotation for the constituent $\beta$ in (46):

$$
\begin{aligned}
& \text { The denotation of } \beta \text { in }(46) \text { for } g\left(D_{1}\right)=\{[>2],[>3],[=3]\} \\
& \llbracket \beta \rrbracket^{g}=[>2] \wedge \neg[>3] \wedge \neg[=3]
\end{aligned}
$$

Now let's bring in the assumption that forced us to adopt (29) in order to prevent (24) from being assigned unattested IIs, viz. the assumption that $[>2]$ is contextually equivalent to $[\geq 3]$ (i.e. to be in a context $c$ such that $[>2] \equiv_{c}[\geq 3]$ ). What we find then is that the denotation we derived in (48) is a contextual contradiction:

19 This follows from the closure conditions and the inability of the alternative-building mechanism to delete occurrences of $K$ (Meyer 2013). 


$$
[>2] \wedge \neg[>3] \wedge \neg[=3] \equiv_{c} \perp
$$

Consequently, the denotation of (46) contextually entails $K \perp$, i.e. an irrational speaker. This contradicts the foundation of conversations governed by the Gricean maxims, namely the assumption of a rational speaker. Therefore, Gricean interlocutors can conclude that $[>3]$ and [=3] cannot both be members of $D_{1}$ in (46) (the domain of the lower exh operator) and hence, given that $R$ cannot break contextual symmetry, that neither can. Therefore, no SIs or IIs are assigned to (48): if $D_{1}$ excludes both [>3] and [=3], $D_{2}$ excludes both $K[>3]$ and $K[=3]$; consequently, the two exh operators apply vacuously. This is a good result since no SIs or IIs are attested for (24).

Next, we contrast what we just derived with what (45) implies for (23), see (50).

$$
\left[\gamma \operatorname{exh}_{D_{2}}\left[\mathrm{~K}\left[\beta \text { two }\left[\lambda_{1}\left[\operatorname{exh}_{D_{1}}\left[\alpha \text { Ann owns at least } t_{1} \text { dogs }\right]\right]\right]\right]\right]\right.
$$

Again, our assumptions are consistent with $g\left(D_{1}\right)$ being the set $\{[\geq 2],[>2],[=2]\}$ (details omitted). However, unlike before, we now observe that the lower exh operator applies vacuously because of the logical facts in (51), which prevent $[>2]$ and $[=2]$ from being excluded by exh.

$$
[\geq 2] \wedge\left\{\begin{array}{lll}
\neg[>2] & \Rightarrow & {[=2]} \\
\neg[=2] & \Rightarrow & {[>2]}
\end{array}\right.
$$

Importantly, the fact that $[>2]$ and $[=2]$ fail to meet the excludability condition of exh doesn't mean that they cannot be members of $D_{1}$ : their membership doesn't lead to an improper meaning assignment as in the previous case. Therefore, $K[>2]$ and $K[=2]$ can be members of $D_{2}$. Consequently, the labeled constituents in (50) can have the following denotations:

The denotation of the labeled constituents in (50)

$$
\begin{array}{lr}
\llbracket \alpha \rrbracket^{g}=[\geq 2] & \quad \text { with } g\left(D_{1}\right)=\{[\geq 2],[>2],[=2]\} \\
\llbracket \beta \rrbracket^{g}=\llbracket \alpha \rrbracket^{g} & \\
\llbracket \gamma \rrbracket^{g}=K[\geq 2] \wedge \neg K[>2] \wedge \neg K[=2] \quad \text { w/g }\left(D_{2}\right)=\{K[\geq 2], K[>2], K[=2]\}
\end{array}
$$

Since $\llbracket \gamma \rrbracket^{g}$ logically entails $I[>2]$ and $I[=2]$, we are able to derive from (50) the IIs attested for (23), the desired result.

The next step in our demonstration is concerned with the examples in (3) and (4), whose structures, as imposed by (45), are given in (53).
a. $\left[\gamma \operatorname{exh}_{D_{2}}\left[\mathrm{~K}\left[\beta\right.\right.\right.$ eight $\left[\lambda_{1}\left[\operatorname{exh}_{D_{1}}\left[\alpha\right.\right.\right.$ Bella weighs at least $\left.\left.\left.\left.\left.\left.t_{1} \mathrm{~kg}\right]\right]\right]\right]\right]\right]$
b. $\left[\gamma \operatorname{exh}_{D_{2}}\left[\mathrm{~K}\left[\beta\right.\right.\right.$ eight $\left[\lambda_{1}\left[\operatorname{exh}_{D_{1}}\left[\alpha\right.\right.\right.$ Bella weighs more than $\left.\left.\left.\left.\left.\left.t_{1} \mathrm{~kg}\right]\right]\right]\right]\right]\right]$ 
How obligatory irrelevance, symmetric alternatives, and dense scales conspire

If the variable $D_{1}$ in (53a) and (53b) is assigned the dense set in the first and second line of Table 1, respectively, then we derive virtually the same result for this pair as for the pair in (50) and (46) with an exhaustification domain exhibiting contextual symmetry. The only difference is that the constituent $\beta$ in (53b) denotes a logical contradiction (Fox \& Hackl 2006; Gajewski 2009) and not a contextual contradiction as in (46). Thus, we derive IIs for (53a) and no IIs for (53b) (in the latter case, if $D_{1}$ is assigned the singleton $\{[>8]\}$ ). If, in contrast, $D_{1}$ in (53a) and (53b) is assigned the non-dense set in the third and fourth line of Table 1, respectively, then we derive for both structures the SIs $\neg[>9]$ and $\neg[=9]$ at the level of the lower exh operator and for (53a) IIs about $[>8]$ and $[=8]$ at the level of the higher exh.

In the same way, if $D_{1}$ in (54a) and (54b) is assigned the non-dense set in the fifth and sixth line of Table 1, respectively, we derive for both structures the SIs $\neg[>200]$ and $\neg[=200]$ at the level of the lower exh operator and for (54a) IIs about $[>100]$ and $[=100]$ at the level of the higher exh.
a. $\left[\operatorname{exh}_{D_{2}}\left[\mathrm{~K}\right.\right.$ [one hundred $\left[\lambda_{1}\left[\operatorname{exh}_{D_{1}}\right.\right.$ [at least $t_{1}$ people attended]]]]]
b. $\left[\operatorname{exh}_{D_{2}}\left[\mathrm{~K}\right.\right.$ [one hundred $\left[\lambda_{1}\left[\operatorname{exh}_{D_{1}}\right.\right.$ [more than $t_{1}$ people attended]]]]]]

Thus, to conclude, we derive all attested SIs and IIs for the sentences under consideration and no unattested SIs or IIs.

\section{Conclusion}

If relevance is assumed to be closed under speaker belief (Fox 2016), then the Maxim of Quantity (MQ) compels speakers to express their epistemic state about every relevant proposition (Buccola \& Haida 2019). This commitment (Strong Epistemic Transparency, or SET), we showed, can predict the contrast in ignorance inferences between at least and more than: the former can logically entail ignorance due crucially to symmetry between formal alternatives, while the latter cannot. To obtain these results, we brought in several auxiliary assumptions, such as the hypothesis that semantic scales are universally dense (UDM; Fox \& Hackl 2006) and the assumption that SET, and hence MQ, involves a logical, rather than contextual, notion of entailment. Although Buccola \& Haida (2019) provide motivation for the latter assumption, we showed here how we might dispense with it, and in the process also capture cases where more than can induce scalar inferences with round numerals (Cummins et al. 2012; Enguehard 2018). Although this extended account no longer relies on SET, it has in common with the SET account that symmetry between formal alternatives is what drives ignorance inferences; that non-strict (cf. class B; Nouwen 2010) modifiers like at least always involve symmetric alternatives, hence entail ignorance; and that strict (cf. class A) modifiers, due crucially to the UDM, never involve (logically) symmetric alternatives, hence cannot entail ignorance. 
Buccola and Haida

\section{References}

Buccola, Brian \& Andreas Haida. 2019. Obligatory irrelevance and the computation of ignorance inferences. Journal of Semantics 36(4). 583-616. doi:10.1093/jos/ffz013.

Büring, Daniel. 2008. The least at least can do. In West Coast Conference on Formal Linguistics (WCCFL), vol. 26, 114-120. Somerville, MA. http://www.lingref. com/cpp/wccfl/26/paper1662.pdf.

Cremers, Alexandre, Elizabeth Coppock, Jakub Dotlačil \& Floris Roelofsen. 2017. Modified numerals: Two routes to ignorance. Manuscript. http: //semanticsarchive.net/Archive/jk2M2Q3O/.

Cummins, Chris, Uli Sauerland \& Stephanie Solt. 2012. Granularity and scalar implicature in numerical expressions. Linguistics and Philosophy 35(2). 135169. doi:10.1007/s10988-012-9114-0.

Enguehard, Émile. 2018. Comparative modified numerals revisited: Scalar implicatures, granularity, and blindness to context. In Semantics and Linguistic Theory (SALT), vol. 28, 21-39. Ithaca, NY. doi:10.3765/salt.v28i0.4403.

von Fintel, Kai \& Irene Heim. 1997. Pragmatics in linguistic theory. MIT classnotes. Fox, Danny. 2007. Free choice and the theory of scalar implicatures. In Uli Sauerland \& Penka Stateva (eds.), Presupposition and Implicature in Compositional Semantics Palgrave Studies in Pragmatics, Language and Cognition Series, chap. 4, 71-120. New York, NY: Palgrave Macmillan. doi:10.1057/9780230210752_4.

Fox, Danny. 2016. On why ignorance might be part of literal meaning. Commentary on Marie-Christine Meyer, MIT Workshop on Exhaustivity. https://drive.google. com/file/d/0B0oeGQ78K8a1Q1BncUVpa1A0eW8/view.

Fox, Danny \& Martin Hackl. 2006. The universal density of measurement. Linguistics and Philosophy 29(5). 537-586. doi:10.1007/s10988-006-9004-4.

Fox, Danny \& Roni Katzir. 2011. On the characterization of alternatives. Natural Language Semantics 19(1). 87-107. doi:10.1007/s11050-010-9065-3.

Gajewski, Jon. 2009. Innocent exclusion is not contradiction free. Manuscript. http:// jon-gajewski.uconn.edu/wp-content/uploads/sites/1784/2016/08/IEnotCF.pdf.

Geurts, Bart \& Rick Nouwen. 2007. At least et al.: The semantics of scalar modifiers. Language 83(3). 533-559. http://www.jstor.org/stable/40070901.

Groenendijk, Jeroen \& Martin Stokhof. 1984. Studies on the semantics of questions and the pragmatics of answers. Amsterdam, Netherlands: University of Amsterdam PhD dissertation. http://hdl.handle.net/11245/1.392528.

Haida, Andreas. 2019. Symmetry, density, and formal alternatives. Snippets 37. 40-42. doi:10.7358/snip-2019-037-haid.

Hintikka, Jaakko. 1962. Knowledge and Belief: An Introduction to the Logic of the Two Notions. Ithaca, NY: Cornell University Press. 
How obligatory irrelevance, symmetric alternatives, and dense scales conspire

Katzir, Roni. 2007. Structurally-defined alternatives. Linguistics and Philosophy 30(6). 669-690. doi:10.1007/s10988-008-9029-y.

Kennedy, Chris. 2015. A “de-Fregean" semantics (and neo-Gricean pragmatics) for modified and unmodified numerals. Semantics and Pragmatics 8(10). 1-44. doi:10.3765/sp.8.10.

Landman, Fred. 2004. Indefinites and the Type of Sets. Malden, MA: Blackwell Publishing.

Lewis, David. 1988. Relevant implication. Theoria 54(3). 161-174. doi:10.1111/j.1755-2567.1988.tb00716.x.

Magri, Giorgio. 2009. A theory of individual-level predicates based on blind mandatory scalar implicatures. Natural Language Semantics 17(3). 245-297. doi:10.1007/s11050-009-9042-x.

Meyer, Marie-Christine. 2013. Ignorance and grammar. Cambridge, MA: Massachusetts Institute of Technology PhD dissertation. http://hdl.handle.net/1721. $1 / 84420$.

Nouwen, Rick. 2008. Upper-bounded no more: The exhaustive interpretation of non-strict comparison. Natural Language Semantics 16. 271-295. doi:10.1007/s11050-008-9034-2.

Nouwen, Rick. 2010. Two kinds of modified numerals. Semantics and Pragmatics 3(3). 1-41. doi:10.3765/sp.3.3.

Nouwen, Rick. 2015. Modified numerals: The epistemic effect. In Luis Alonso-Ovalle \& Paula Menéndez-Benito (eds.), Epistemic Indefinites: Exploring Modality Beyond the Verbal Domain Oxford Linguistics, chap. 11, 244-266. Oxford, England: Oxford University Press. doi:10.1093/acprof:oso/9780199665297.003.0011.

Rooth, Mats. 1985. Association with focus. Amherst, MA: University of Massachusetts Amherst $\mathrm{PhD}$ dissertation.

Schwarz, Bernhard. 2016. Consistency preservation in Quantity implicature: The case of at least. Semantics and Pragmatics 9(1). 1-47. doi:10.3765/sp.9.1.

Spector, Benjamin. 2015. Why are class B modifiers global PPIs? Disjunctions as a model for class B modifiers. Talk presented at the workshop 'New Directions in Negation and Polarity', The Hebrew University of Jerusalem.

Spector, Benjamin. 2018. Revisiting weak exhaustivity. Talk presented at the workshop 'Meaning in non-canonical questions', University of Konstanz.

Westera, Matthijs \& Adrian Brasoveanu. 2014. Ignorance in context: The interaction of modified numerals and QUDs. In Semantics and Linguistic Theory (SALT), vol. 24, 414-431. Ithaca, NY. doi:10.3765/salt.v24i0.2436. 


\author{
Brian Buccola \\ B404 Wells Hall \\ 619 Red Cedar Road \\ Michigan State University \\ East Lansing, MI 48824 \\ buccola@msu.edu \\ Andreas Haida \\ The Edmond and Lily Safra Center for Brain Sciences \\ The Suzanne and Charles Goodman Brain Sciences Building \\ Edmond J. Safra Campus \\ The Hebrew University of Jerusalem \\ Jerusalem, 9190401 \\ andreas.haida@gmail.com
}

